\title{
Vaccination with endosomal unknown epitopes produces therapeutic response in rheumatoid arthritis patients and modulates adjuvant arthritis of rats
}

Innocenzo Caruso ${ }^{1 *}$, Salvatore Santandrea ${ }^{2}$, Mariarita Gismondo ${ }^{3}$, Alessandra Lombardi ${ }^{4}$, Franco Montrone ${ }^{5}$, Enzo Massimo Caruso ${ }^{6}$ and Piercarlo Sarzi Puttini ${ }^{7}$

\begin{abstract}
Background: Our previous results showed that intrasynovial Rifamycin SV caused the lysis of synoviocites and freed the autoantigens which in turn stimulated the immunoregulatory rather than autoreactive $T$ cell response in rheumatoid patients. Here, we hypothesize that disruption in vitro of peripheral blood mononuclear cells, by freeze/thawing or by lytic action of Rifamycin SV, would induce the release of endosomal pathogenic autoantigens from APCs present in the circulation, which could then be isolated from degrading enzymes by ultrafiltration.
\end{abstract}

Methods: The preparation of the ultrafiltrates are based on the rupture of PBMCs $\left(5 \times 10^{6} \mathrm{cells} / \mathrm{mL}\right)$ by the addition of Rifamycin SV in culture $(250 \mu \mathrm{g} / \mathrm{mL})$, which causes the lysis of $90 \%$ of the cells in $3 \mathrm{~h}$, or by three cycles of freeze/ thawing of the PBMC, from $-80^{\circ} \mathrm{C}$ to room temperature. The lysate and the fragmented cells were then centrifuged and ultrafiltered by passage through a filtration device with a cut-off of $10 \mathrm{kDa}$. Also the synovial fluid was subjected to ultrafiltration.

Results and conclusions: At clinical monitoring of the 30th day, 22/58 (38\%) patients subcutaneously treated with the autologous ultrafiltrate prepared by the freeze/thawing of PBMCs reached an ACR20. Comparable results were obtained with the other two ultrafiltrates.

Cell cultures The addition of ultrafiltrates to rheumatoid PBMCs cultures led to the upregulation of a marker for T-regulatory cells, and downregulation of a cell proliferation marker; changes that together have the meaning of a global immunomodulatory response and that only a specific antigen (ultrafiltrate UF-f/t) might induce in the rheumatoid patient, probably by activating pre-existing protective network.

Experimental arthritis All the ultrafiltrates except that prepared by Rifamycin SV were able to modulate the adjuvant arthritis in rats. In particular, longlasting synovial fluid induced a significant reduction of the severity of subsequent arthritis ( $p<0.01$ ) while SF from recent RA effusion (5-10 days after a previous complete extraction) and knee osteoarthrosis were ineffective. It is reasonable to assume there are at least two unknown endosomal immunoactive epitopes; one developing its immunotherapeutic property in RA, and the other, related to the molecule of HSP60, reduces the severity of oncoming arthritis. Both epitopes are present in humans, have a molecular weight of $\leq 10 \mathrm{kDa}$ and do not appear to be bystander antigens. Please see Additional file 1 for the abstract in Italian.

Keywords: Ultrafiltrate from PBMCs, Autologous immunotherapy, Arthritogenic epitope, Immunotherapeutic vaccine, Bystander antigen, Bystander immunotherapy

\footnotetext{
*Correspondence: innocenzo.caruso@tiscali.it

1 Past Chief Rheumatology Unit, L. Sacco Hospital Milano Italy, Corso Italia

11, 20122 Milan, Italy

Full list of author information is available at the end of the article
} 


\section{Background}

Rifamycin SV is an old antibiotic endowed with a cytolytic property that completely lyses synovial cells and most likely frees the autoantigen anchored on the binding groove of antigen presenting cells (APCs) [1, 2]. Rifamycin SV also provokes the release of all short proteins at various stages of enzymatic degradation from the endosomal compartment. The good therapeutic response to Rifamycin SV injected into the arthritic knee [3-7] and, in particular, the systemic effects obtained when the application is extended to many joints [8-10] have led us to hypothesize that this antibiotic, once infiltrated in joints, frees the pathogenic peptide from synovial APCs, which, in conjunction with MHC class II molecules, would otherwise present this peptide to autoreactive $\mathrm{T}$ cell receptors, contributing to the continued immune response. The peptide itself might then instead favor an interaction with the antigen-binding site of an anti-idiotype T-cell (paratope) [11, 12], activating an immunoregulatory response.

The idiotype-anti-idiotype network on membrane is considered central in immunoregulation involving autoantigens [13].

The use of a specific autoantigen at appropriate doses to bypass interaction with autoreactive $\mathrm{T}$ lymphocytes and thus detrimental immune response has been shown to cure spontaneous autoimmune disease, as with glutamic acid decarboxylate (GAD 65) in the non-obese diabetic rat [14]. Thus, the isolation and identification of autoantigens for other autoimmune disorders such as rheumatoid arthritis (RA) might have considerable therapeutic benefit. To facilitate this, the use of peripheral blood mononuclear cells (PMBCs) of subjects with RA as a source to extract the pathogenic peptides in vitro instead of the less accessible synovial cells has been considered.

This is based on the notion that, although very different in number, many peripheral blood cells including macrophages, dendritic cells, and B lymphocytes, possess functional characteristics that are commonly attributed to actual APCs $[15,16]$. All potential APCs are capable of degrading the antigens in their endosomal compartment; the subsequent transfer of the resultant peptides to the cell surface and their presentation to lymphocytes gives rise to immunoinflammation [17].

Within PBMCs, research on the pathogenesis of systemic and organ-specific autoimmune diseases has expanded the complex role of B cells from the synthesis of antibodies and pro-inflammatory cytokines to that of internalization and presentation of antigens $[18,19]$.

$\mathrm{B}$ cells process and present the antigen peptides to $\mathrm{CD} 4+\mathrm{T}$ lymphocytes in the context of MHC class II alleles. It has been argued that in established autoinflammatory conditions B cells represent a potent reservoir of APCs for activating T cells [20].

Starting from these considerations, we thought of inducing the release of pathogenic peptides from PBMCs in vitro by causing the lysis or fragmentation of cells through the use of Rifamycin SV as a cytolytic substance, or through freeze/thawing of cells, respectively. Separation of unknown PBMC substances in the tube could determine the leak of autoantigen as well as all the intracellular material in which there are many unknown substances.

The separation of peptides and any other substances from lysates and from the suspension of cell fragments could be obtained by centrifugation and subsequent immediate ultrafiltration $(\mathrm{mw} \leq 10 \mathrm{kDa})$. All the molecules of low weight could be collected in the flowthrough. Among these, in addition to the short proteins from the endosomal compartment, might be included the specific arthritogenic peptides.

The same method of separation could be applied to rheumatoid synovial fluid as well. In the context of synovitis, the release of pathogenic peptides is induced by inflammatory disruption of the synovial cells. The immediate ultrafiltration of centrifuged fluid would allow immunoactive peptides, free of molecules involved in the endosomal processing of antigens (e.g., class II-associated invariant peptide) and not linked to the molecule HLA DR, to be collected in the flow-through.

As first step in determining the identity of the autoantigen(s) in RA, in this study, we have prepared four ultrafiltrates, two of which were derived from the breakdown of PBMCs, one from the supernatant of PBMCs cultures and one from synovial fluid.

Three of these were utilized in trials of autologous immunotherapy by injecting them subcutaneously in patients with early RA (disease duration of less than 12 months) and for a protection protocol in adjuvant arthritis (AA) in rats (iv administration 7, 8 days after AA induction). We chose AA model because is considered a disease mediated by auto reactive $T$ cells cross-reacting with mycobacterial hsp65 [21, 22], which is a stress protein highly conserved through the evolution, from bacteria to man, and yet the arthritogenic autoantigen(s) remains elusive [23].

\section{Methods}

\section{Preparation of ultrafiltrates}

All procedures were performed in BSL3. The ultrafiltrate preparation protocol was performed using sterile equipment in laminar flow cabinet with HEPA filter. The sterility of PBMC ultrafiltrates was tested by placing it in culture on Blood Agar and Muller Hinton broth. 


\section{Isolation of peripheral blood mononuclear cells}

Peripheral blood mononuclear cells were isolated from whole blood using Histopaque-1077 polysucrose and sodium diatrizoate solution, according to manufacturer's instructions

Briefly, whole blood samples from the patients were labeled with a univocal barcode defined by the protocol and diluted 1:2 with $1 \times$ PBS in a labeled 50-mL Falcon tube. In a second Falcon tube, $10 \mathrm{~mL}$ of Histopaque-1077 was added, onto which $20 \mathrm{~mL}$ of diluted blood was subsequently layered. Then, Falcon tubes were centrifuged at $750 \times g$ for $30 \mathrm{~min}$ at room temperature, without brake deceleration. The PBMCs were then collected, divided into four $15-\mathrm{mL}$ Falcon tubes, and centrifuged three times at $650 \times g$ for $10 \mathrm{~min}$, at room temperature, in $10 \mathrm{~mL}$ of $1 \times$ PBS solution to remove the red blood cells. Finally, PMBCs were counted and stored at $-80{ }^{\circ} \mathrm{C}$, at a final concentration of $5 \times 10^{6}$ cells $/ \mathrm{mL}$, in tubes labeled with the appropriate protocol barcode.

\section{Ultrafiltrate UF-S (mononuclear cell supernatant)}

PBMCs $\left(5 \times 10^{6}\right.$ cells $\left./ \mathrm{mL}\right)$ were cultured in shell vials in RPMI 1640 medium containing $20 \%$ fetal calf serum, at $37{ }^{\circ} \mathrm{C}$, in a $5 \% \mathrm{CO}_{2}$-enriched atmosphere for $3 \mathrm{~h}$. At the end of the incubation period, the supernatant was collected after centrifugation at $350 \times g$ for $10 \mathrm{~min}$, ultrafiltrated by passage through a Millipore filtration device with a molecular weight cut-off value (MWCO) of $10,000 \mathrm{kDa}$ (Millipore Corporation, Bedford, USA), and stored at $-20^{\circ}$ until use.

\section{Ultrafiltrate UF-R}

For the preparation of ultrafiltrate for use in immunotherapy, rifamycin SV, stored in 10-mL (250 mg) vials (Lepetit Spa, Milan) (for intravenous use), was utilized.

PBMCs $\left(5 \times 10^{6}\right.$ cells $\left./ \mathrm{mL}\right)$ were cultured under the same conditions, but in the presence of $250 \mu \mathrm{g} / \mathrm{mL}$ of rifamycin SV, for $3 \mathrm{~h}$. At the end of the incubation period, the rate of cell lysis observed was $90 \%$. The supernatant was collected after centrifugation at $350 \times g$ for $10 \mathrm{~min}$ and the ultrafiltrate was prepared as described above. The flow-through was used within 2-3 days, and the remainder was stored at $-20{ }^{\circ} \mathrm{C}$. The ultrafiltrate prepared with rifamycin SV was used in clinical trials.

\section{Ultra filtrates UF-f/t}

PBMCs were purified using Histopaque-1077, as described above, but cell lysis was not performed.

Briefly, PBMCs were subjected to three freeze/thaw cycles from $-80{ }^{\circ} \mathrm{C}$ to room temperature and centrifuged at $1500 \times g$ for $10 \mathrm{~min}$; up to $1.5 \mathrm{~mL}$ of sample was then transferred into Millipore filtration devices with a MWCO of $10,000 \mathrm{kDa}$, and centrifuged at $1500 \times \mathrm{g}$ for
10 min in labeled 50-mL Falcon tubes. The ultrafiltrate was collected in $2 \mathrm{~mL}$ tubes and stored at $-20^{\circ} \mathrm{C}$.

It should be noted that epitopes bound to HLA-DR $(>10 \mathrm{kDa})$ are too large to pass through the pores of the ultrafilter while the free pathogenic epitope and other endosomal peptide easily pass.

Generally, the ultrafiltrate was prepared a few days before its subcutaneous administration. A portion of the ultrafiltrate was stored separately for in vitro studies (see below).

\section{Ultrafiltrate UF-sf}

Synovial fluid from a long-lasting (for at least a month) inflammatory effusions, was centrifuged to remove the cells and then subjected to ultrafiltration using the procedures described above. The ultrafiltrate was used within 2 days, and the remainder was stored as above.

\section{Rheumatoid arthritis \\ Patients}

Patients recruited for this study fulfilled the American College of Rheumatology (ACR) 1987 revised criteria for RA [24]; specifically, patients with disease duration of less than 1 year and who had not been previously treated with immunosuppressive agent were recruited. The inclusion criteria were as follow: age between 17 and 75 years, ACR functional class I, II or III [25], presence of active disease symptoms such as nine or more tender joints at rest or in motion, six or more swollen joints, a pain score of greater than four on the Visual Analog Scale (VAS) and an erythrosedimentation rate (ESR) of $>30 \mathrm{~mm} / \mathrm{h}$. The exclusion criteria were as follow: known allergy to Rifamycin SV antibiotic, a positive intra-dermal sensitivity test for Rifamycin SV, the intra-articular or parenteral administration of corticosteroids 4 weeks prior to the study.

\section{Study design and monitoring}

In this study, three of four ultrafiltrate samples, which were prepared as described above, were used: UF-sf (prepared from rheumatoid acellular synovial fluid), UF$\mathrm{f} / \mathrm{t}$ (prepared by freeze/thawing of PBMCs), and UF-R (obtained by lysing mononuclear cells from patients using Rifamycin SV).

The objectives of this study were to investigate the efficacy of autologous ultrafiltrates, administered via subcutaneous injection at baseline on two consecutive days, and to compare the response rate on the 10th day vs those on the 30th day in each of the three treatment groups.

AS the groups were not homogeneous in terms of patient characteristics the response rates of the different groups to treatment could not be compared, therefore the groups were evaluated separately. 
The choice of non-placebo group was based on ethical, practical and methodological considerations. Moreover, a single blind study design, in which the investigator evaluating the response was not aware of the treatment administered, ensured the absence of assessment bias.

The study design included randomization between groups; however after the first ten randomized patients $70 \%$ of patients tested positive for intra-dermal sensitivity test to Rifamycin SV (wheal diameter $\geq 0.5 \mathrm{~cm}$ ). This unexpectedly high percentage of patients sensitive to Rifamycin SV necessitated a change in the design of the study, in that the randomization procedure was no longer considered feasible.

The study design was modified according to the following criteria: patients (a) with a previous or current history of allergic or autoimmune diseases other than RA, such as atopic dermatitis, chronic hypersensitivity pneumonitis, leukopenia due to hypersensitivity, and asthma, (b) with clinical conditions that may be aggravated by Rifamycin SV such as elevation of liver enzymes, impaired creatinine clearance, cholelithiasis, (c) who may have been treated with the Rifamycin SV (e.g., patients with previous tubercular infection) and those who were treated with this antibiotic via the intrasynovial route for arthritis, and (d) nurses were excluded from the UF-R group and included in the UF- $\mathrm{f} / \mathrm{t}$ group. In addition, only patients presenting synovial effusion of knees were included in the UF-sf group.

A total of 115 patients with active RA were enrolled in this study at the L. Sacco Hospital, 11 of whom had joint effusions of both knees. Patients in groups UF-R and UF$\mathrm{f} / \mathrm{t}$ were administered the previously prepared ultrafiltrate $(1.7-2 \mathrm{~mL})$ at subcutaneous sites on the forearm on two consecutive days, whereas those in the UF-sf group received $0.8-2 \mathrm{~mL}$ of ultrafiltrate upon availability. An adverse reaction to these antigens was unlikely as peripheral lymphocytes reactive to self peptides are eliminated during ontogeny via negative selection.

The study design was approved by the Ethics Committee of L. Sacco Hospital and by the Ethics Committee of the Regional Government of Lombardia, Italy (Resolution number 599 of July 30 2009). Informed consent was obtained from all patients. Patients underwent a complete physical assessment and $30-50 \mathrm{~mL}$ peripheral blood was drawn for blood tests and cell ultrafiltrate preparations and synovial fluid, where present, was completely drained. The following were evaluated for measurement of changes from the baseline at every assessment: swollen joint count, tender joint count, pain score (VAS), patient and physician global assessment of disease activity (VAS), and efficacy according to the ACR criteria [26]. Inspection of the injection site and general tolerability were evaluated at the 10th and 30th day of the clinical trial.
An immune response to a subcutaneous administration of any antigen rarely lasted for more prolonged than 30 days. However, more detailed studies are necessary to define the schema of this immunotherapy.

\section{Rheumatoid arthritis—in vitro studies Cells and ultrafiltrate}

The cells for the cultures were obtained from a pool of about $50 \mathrm{~mL}$ whole blood, drawn from six patients with active rheumatoid disease. The cells, purified on Histopaque-1077 were frozen at $5-10 \times 10^{6} \mathrm{cells} / \mathrm{mL}$ and stored in a cryogen tank until use. At the time of use, the PBMCs were thawed and centrifuged at $750 \mathrm{~g}$ for $10 \mathrm{~min}$, washed $3 \times$ with PBS, and resuspended at $0.5-2 \times 10^{6}$ cells/mL DPBS (Dulbecco's Phosphate-Buffered Saline. Carlo Erba. C Dasit group. Milan).

Cells were grown in RPMI1 1640 supplemented with $10 \%$ heat inactivated human $\mathrm{AB}$ serum, $100 \mathrm{U} / \mathrm{mL}$ penicillin, $100 \mu \mathrm{g}$ streptomycin, and $1 \mathrm{mM}$ glutamine. The PBMCs were cultured at $0.5-2 \times 10^{6}$ per well in a 48 well plate for $48 \mathrm{~h}$ with media alone, or stimulated with plastic bound anti-CD3 (clone OKT3) $(5 \mu \mathrm{g} / \mathrm{mL})$ and soluble anti-CD28 $(2 \mu \mathrm{g} / \mathrm{mL}), 10 \mu \mathrm{g} / \mathrm{mL}$ tetanus toxoid-derived peptide (QYIKANSKFIGITE) or hsp60 (GEALSTLVVNKIRGT) (Becton-Dickinson). Cultures were set up in duplicate with one set receiving $400 \mu \mathrm{L}$ ( $0.1 \mathrm{mg}$ protein per well) of UF-sf. The PBMC ultrafiltrate was adjusted to $10 \%$ human $\mathrm{AB}$ serum prior to addition to cell cultures. After $48 \mathrm{~h}$, the cells were harvested and immunostained as described below. The UF-sf for the in vitro studies were the same as those prepared and employed as autologous vaccines.

\section{Ultrafiltrates}

For UF-sf, the samples stored at $-20^{\circ} \mathrm{C}$ were thawed and pooled in the laboratory for in vitro assays. A micro BCA protein assay (Pierce Biotecnology, Rockford, IL) was performed to determine the protein concentration of the pooled ultrafiltrates. The ultrafiltrate prepared with Rifamycin SV was excluded from this study because it cannot be removed from the extract, making in vitro studies difficult.

\section{SDS-PAGE and western blot analysis}

Several experiments were performed to attempt to visualize the protein of interest on a gel. These experiments were performed with some of the patient samples as well as with samples from donors recruited for this purpose. The ultrafiltrates prepared with Rifamycin SV were run on 4-12\% SDS-PAGE gels and silver staining and western blot analysis with anti-hsp60 (Becton-Dickinson, San Diego, CA), as the detection antibody, were used for protein visualization. 


\section{FACS analysis}

Cell staining was used to measure (1) TNF-alpha production by T cells and macrophages, (2) CD69 as an indicator of cell proliferation and (3) CTLA-4 expression by CD4+/CD25+ cells as an inhibitor of $\mathrm{T}$ cell function. Isotype controls were included to determine non-specific antibody staining. Monensin was added to the PBMC cultures $4 \mathrm{~h}$ prior to immunostaining, and the cells were harvested and extracellular immunostaining was performed with antibodies to human CD3 (clone UCHT1), CD4, CD25, CD69 and CD11 (Becton-Dickinson). The cells were fixed with paraformaldehyde and intracellular staining was performed with antibodies to TNF-alpha and CTLA- 4 in the presence of saponin. Appropriate isotype controls were also included.

\section{Ultrafiltrate from a normal donor is not toxic to normal BPMCs}

In order to test the possible toxicity of ultrafiltrate on cells and to determine the appropriate volume to use for subsequent patient studies, the effects of normal donor ultrafiltrate on CD69 expression, a marker for cell proliferation, by $\mathrm{T}$ cells in cultures with normal PBMCs, were evaluated. PBMCs were cultured with increasing volumes of UF-f/t (from 0 to $500 \mu \mathrm{L}$ ) in the presence or absence of anti-human CD3 $(5 \mu \mathrm{g} / \mathrm{mL})$ and CD28 $(2 \mu \mathrm{mL})$. Cells were incubated for 24 or $48 \mathrm{~h}$ and co-stained with antiCD3 and anti-human CD69. The proliferation of stimulated cells, as measured by the expression of CD69, (\% CD69/CD3), was consistently high with all volumes of ultrafiltrate.

Not being toxic the maximum feasible amount of ultrafiltrates $(400 \mu)$ were used. Additionally, CD69 expression was higher at $48 \mathrm{~h}$ and that cultures time was used (the tables are available on request).

\section{Adjuvant arthritis in rats \\ Animals studies were performed at the LITA laboratories, Microbiology Chair, University of Milano (Italy)}

Induction and assessment of arthritis Adjuvant arthritis (AA) was induced by intracutaneous injection of $0.1 \mathrm{~mL}$ of paraffin oil containing $10 \mathrm{mg} / \mathrm{mL}$ of heat-killed Mycobacterium butirricum, into the base of the tail of female Lewis rats. Active disease developed 11-12 days after injection reaching a peak at 17-21 days; this was followed by slow resolution. Severity of arthritis was evaluated every fourth day by the measurement of clinical signs in each paw, on a scale of 0-2. The maximum arthritis score was eight per animal. The rats lost $5 \%$ of their body weight over the course of the clinical disease.

Protection protocol using ultrafiltrates Fifty rats were randomized into the following five treatment groups:
Rifamycin SV alone $(250 \mu \mathrm{g} / \mathrm{mL})$, saline solution, UF-S, UF-f/t or UF-R, and intravenously treated, with $0.5 \mathrm{~mL} /$ rat/day, on days 7 and 8 after AA induction. We did not administer ultrafiltrate during the overt manifestation of arthritis.

Collection of synovial fluid Synovial fluids were obtained from eight patients with rheumatoid arthritis with longlasting knee effusions, six with recent effusions (5-10 days after a previous complete extraction) and six with arthrosis. Synovial fluids were centrifugated at $400 \times g$ for $15 \mathrm{~min}$; the supernatant was the ultrafiltrated as above and stored at $-20^{\circ} \mathrm{C}$ until use.

Protection protocol using ultra filtrate UF-sf Animals $(\mathrm{n}=8)$ were injected in the tail vein with $0.5 \mathrm{~mL}$ of UF-sf or saline solution (control) (8). Each animal was treated with UF-sf obtained from an individual patient. Twentyone days after AA induction the arthritis severity was scored by grading each hind paw.

\section{Statistical analysis \\ In vitro studies}

Changes from the 10th to the 30th day were assessed using a Chi square test for the UF-f/t and UF-R groups using $95 \%$ confidence intervals, whereas the Fisher's exact test was used for the third comparison with the UF-sf group, because of the low number of patients therein. For immunohistochemistry, nonspecific staining as determined with the isotype controls was subtracted (as percentage) from the positive responses. Differences between matched untreated PBMCs and samples treated with ultrafiltrates were analyzed by the Wilcoxon paired rank test.

\section{Clinical studies}

All data concerning the protective protocol in rats were analysed with non-parametric tests. To compare the different groups, the Friedman's test was performed with time and treatment as variables. Synovial fluid results were analysed by one way analysis of variance followed by Bonferroni $\mathrm{T}$ test for multiple comparisons.

\section{Results and discussion}

\section{Rheumatoid arthritis}

The results reported in Table 1 show that the three autologous ultrafiltrates, administered subcutaneously, led to good therapeutic effects. In some patients, these effects occurred early, sometimes after just a week, and lasted at least until the final check of the 30th day. In the UF-R group, $37 \%(17 / 46)$ of the patients achieved an ACR20 by the 10 th day vs $33 \%(19 / 58)$ of those receiving UF$\mathrm{f} / \mathrm{t}$. The patients who received UF-sf showed a still higher 
Table 1 Disease activity score after subcutaneous injection of autologous ultrafiltrates in early rheumatoid patients

\begin{tabular}{|c|c|c|c|c|c|c|c|}
\hline \multirow[t]{2}{*}{ Ultrafiltrates $^{a}$} & \multirow[t]{2}{*}{ Pts } & \multicolumn{2}{|c|}{$A C R \geq 20^{b}$ (10th day) vs (30th day) } & \multirow[t]{2}{*}{$\mathbf{R R}^{\circ}$} & \multirow[t]{2}{*}{$95 \%$} & \multirow[t]{2}{*}{$\mathrm{Cl}$} & \multirow[t]{2}{*}{$\mathrm{p}$ value } \\
\hline & & (\%pts10th day) & (\%pts 30th day) & & & & \\
\hline UF-R rifamycin SV (from $5 \times 10^{6}$ cells $/ \mathrm{mL}$ ) & 46 & $17(37)$ & $19(41)$ & 0.90 & 0.54 & 1.49 & NS \\
\hline Ul-f/t freeze/taw (from $5 \times 10^{6}$ cells $/ \mathrm{mL}$ ) & 58 & $19(33)$ & $22(38)$ & 0.86 & 0.53 & 1.42 & NS \\
\hline UF-sf (from acellular sin. fluid) & 11 & $7(64)$ & $7(64)$ & 1 & 0.53 & 1.88 & - \\
\hline
\end{tabular}

a One subcutaneous administration in two consecutive days. For doses, see text

b American College Rheumatology criteria $\geq 20^{\circ}$ relative risks (RR), calculated using Chi square Pearson test

ACR20 response rate of $64 \%(7 / 11)$. In the subsequent 20 days, the number of patients with a positive therapeutic response increased slightly in UF-R and UF-f/t, from 17 to 19 (41\%) and 19 to 22 (38\%), respectively, and the final results were comparable between these groups. In contrast, patients in the UF-sf group remained unchanged (64\%). The comparison of the results between the 10th and the 30th day did not show any significant difference in any of the three groups.

Among the three ultrafiltrates, UF-f/t would appear to be preferred for clinical use although its response rate by the 30th day was the lowest $(22 / 58,38 \%)$, because it is the easiest to prepare and can be prepared and administered the same morning. On the other hand, UF-R has the disadvantage of potential patient hypersensitivity reaction to the antibiotic, Rifamycin SV, which cannot be eliminated from the PBMC lysates (see safety). It has been reported that serious side effects occur in patients who take the Rifamycin SV intermittently [27]. Finally, UF-sf presents the greatest obstacle for practical use because only a minority of patients have abundant synovial fluid in their knees.

\section{Rheumatoid arthritis in vitro studies}

As previously suggested, we expect that the immuneactive epitope should be the same between the three ultrafiltrates. Therefore, for the in vitro studies, we employed the ultrafiltrates UF-f/t and UF-sf but not UF-R, as it was prepared with Rifamycin SV.

Notably, in the latter the Rifamycin SV itself was the only low molecular weight protein detected (SDS-PAGE), which might have interfered with the detection of other small molecular weight proteins. The Western blots also showed no proteins when an anti-heat shock protein antibody was used for detection.

\section{Immunomodulatory activity of ultrafiltrates on PBMCs from patients with RA}

For this study we chose to test the ultrafiltrate UF-sf because the concentration of the pathogenic epitope in synovial fluid could be greater than in the ultrafiltrate UF-f/t.

This we have deduced from experiment reported in Table 4 (see below) in which longlasting synovial fluid was significantly more effective than the recent one in the inhibitory activity against AA showing the ability of the pathogenic epitope to accumulate.

PBMCs $\left(1 \times 10^{6}\right.$ per well $)$ patients with active RA (6) were cultured in duplicate with UF-sf and stimulated with anti-CD3/CD28. After $48 \mathrm{~h}$, the cells were harvested and immunostained for CD4/CD25, CTLA-4, CD69, CD11c and TNF-alpha. Very little TNF-alpha expression resulting from any of the treatments (peptides) was identified. Only anti-CD3 and CD28 stimulated the cells to proliferate and this proliferation as measured by CD69 was significantly decreased by the addition of ultrafiltrate ( $\mathrm{p}=0.04$ ) (Fig. 1). CTLA-4 expression (a marker of $\mathrm{T}$ regulatory cells) by the same cell population increased slightly (not shown) despite the decrease in CD69 expression and was significant in the case of CD4+CD25+ T cells $(p=0.05)$ (Fig. 2). For background correction, nonspecific staining as determined using the isotype controls, was subtracted from the positive responses.

\section{Immunological response to autologous PBMC ultrafiltrates in patients with RA previously vaccinated (30 days) with autologous UF-f/t}

PBMCs from one normal subject and three patients with early RA who had been previously vaccinated, i.e., subcutaneously treated with autologous UF-f/t, were

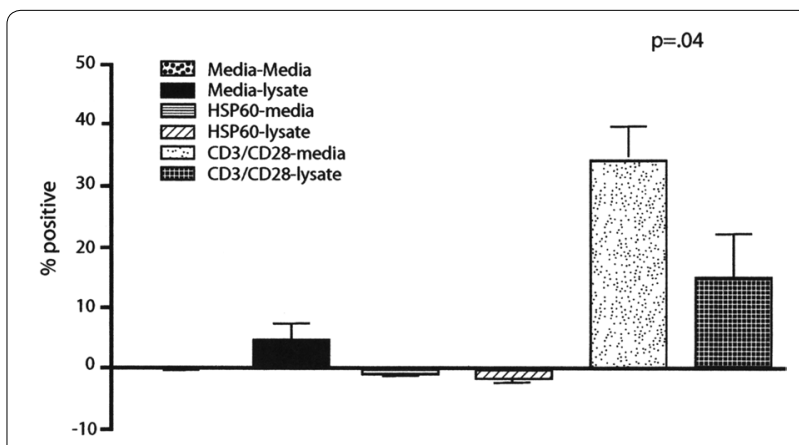

Fig. 1 Expression of CD69 by CD4+CD25+ cells from six RA patients. Results are shown as the mean and standard deviation of the six patients. Only anti-CD3 and CD28 stimulate the cells to proliferate and this proliferation as measured by CD69 was significantly decreased by the addition of lysate 


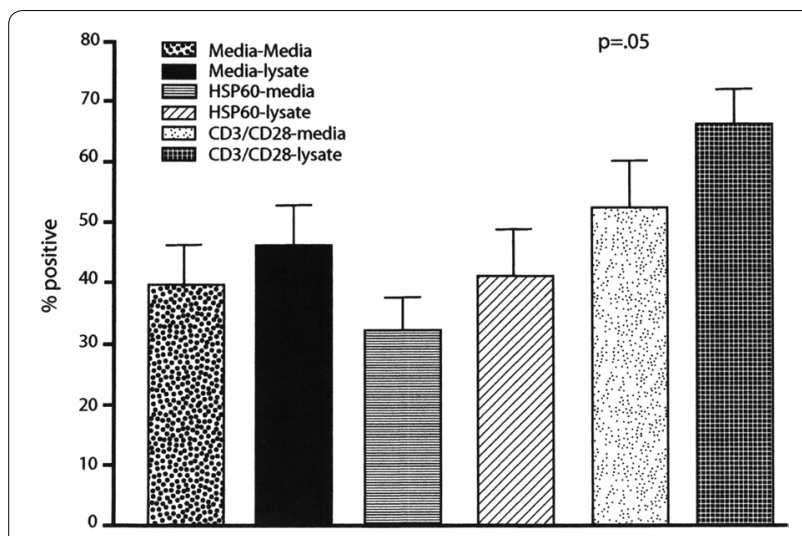

Fig. 2 Expression of CTLA-4 by CD4+CD25+ cells from six RA patients. Results are shown as the mean and standard deviation of six patients. CTLA-4 by the same cell population increase slightly despite the decrease in CD69 expression (not shown) and was significant in the case of the CD4+CD25+T cells

cultured with the same autologous ultrafiltrate and then immunostained. When the Wilcoxon paired samples test was used to determine the differences between no treatment and subcutaneously treatment with autologous ultra filtrates, no statistically significant differences were observed for any of the markers studied (Table 2). However, when CTLA-4 expression by CD4+CD25+ T-cells was examined, the addition of ultrafiltrate decreased the level of this marker in normal donors $(-33 \%)$, which appeared to correlate with the decrease in CD69+ expression. The opposite effect was observed in the autologous vaccinated patients with RA, who exhibited a decrease in CD69+ by CD4+CD25+ $\mathrm{T}$ cells but no change in CTLA-4 expression by the same cells following the addition of ultrafiltrate. In addition, there was little TNF-alpha production by either T cells or monocytes/ macrophages.

\section{Synthesis}

(a) The addition of UF-sf to PBMCs cultured from nonvaccinated patients with RA (6) elicited significant

\begin{tabular}{|c|c|c|c|c|}
\hline Donor & $\begin{array}{l}\text { \% CD69+/ } \\
\text { CD4+ }\end{array}$ & $\begin{array}{l}\% \text { CD69+/ } \\
\text { CD4+CD25+ }\end{array}$ & $\begin{array}{l}\% \text { CTLA- } \\
4+/ C D 4+\end{array}$ & $\begin{array}{l}\text { \% CTLA-4+/ } \\
\text { CD4+CD25+ }\end{array}$ \\
\hline $\begin{array}{l}\text { Normal } \\
\qquad(n=1)\end{array}$ & -25 & -18 & +4 & -33 \\
\hline $\begin{array}{l}\text { Patients } \\
\quad(n=3)\end{array}$ & -7 & -15 & +4 & +3 \\
\hline
\end{tabular}

The results are shown as the untreated cells minus cells plus lysate. CD69 and CTLA-4 were down-regulated in the normal donor reduction of $\mathrm{CD} 69$ by $\mathrm{CD} 4+$ cells $(\mathrm{p}=0.04)$ and at the same time an increase of CTLA-4 expression by CD4 + CD25+ cells $(p=0.05)$.

(b) The addition of autologous UF-f/t to PBMCs cultured from vaccinated patients elicited minimal change in cellular markers.

(c) The addition of autologous UF- $f / t$ decreased the expression of both CD69 (-25\%) and CTLA-4 $(-33 \%)$ in normal subjects.

(d) The addition of antigen p1 (HSP65) did not show any response.

(e) Addition of the ultrafiltrate did not elicit a pro inflammatory response in any of the studied subjects: normal donor, patients with RA, or patients with RA previously vaccinated with autologous PBMC ultrafiltrate.

\section{Adjuvant arthritis}

Table 3 reports the clinical scores of AA. Prevention of paw swelling after treatment with UF-S and UF-f/t was statistically significant $(\mathrm{p}<0.01)$ compared with the control groups. These results showed that active unknown epitopes would be released in UF-S even during $3 \mathrm{~h}$ of culture (in the absence of Rifamycin), following a partial spontaneous cytolysis. On the contrary, AA was not inhibited in rats treated with UF-R and Rifamycin alone (Table 3) and also by the UF-sf from patients with arthrosis (Table 4). Arthrosis is commonly accepted as the normal control for RA.

The administration of long-lasting (more than a month) RA synovial fluid, UF-sf, induced a significant reduction of the severity of subsequent arthritis in rats $(\mathrm{p}<0.01)$ while SF from recent RA effusion $(5-10$ days after a previous complete extraction) was ineffective (Table 4). The ineffectiveness of the recent fluid is probably due to the gradual accumulation over time of immunoactive epitopes from lysis of proliferating synoviocites and deeper mononuclear cells in inflamed synovium. The control saline treated animals had a severe arthritis, as demonstrated by the high clinical scores (Table 4).

In conclusion, longstanding synovial fluid, UF-sf, the supernatant of PBMC cultures, UF-S, and Ultrafiltrate of PBMCs fragmented with freeze/thawing, UF- $f / t$, all from subjects with $\mathrm{RA}$, are able to modulate the immune mechanism implicated in AA and to stimulate protective immunity.

In Table 5, we summarize the results of autologous immunotherapy in RA patients and the protection profile of the human ultrafiltrates administered in rats with oncoming arthritis.

Our strategy to extract and identify pathogenic epitopes was linked to the notion that in a systemic disease such as RA, the epitope would be present in the 
Table 3 Modulation of adjuvant arthritis development in rats by administration of ultrafiltrates obtained from PBMCs of RA patients

\begin{tabular}{|c|c|c|c|c|c|c|c|c|c|c|}
\hline \multirow[t]{2}{*}{ Days from Al } & \multicolumn{2}{|l|}{ UF-S** } & \multicolumn{2}{|l|}{$U F-f / t^{* *}$} & \multicolumn{2}{|l|}{ UF-R } & \multicolumn{2}{|l|}{ Rifamycin } & \multicolumn{2}{|l|}{ Saline sol. } \\
\hline & AA score & Median & AA score & Median & AA score & Median & AA score & Median & AA score & Median \\
\hline 11 & $\begin{array}{l}00000 \\
00233\end{array}$ & 0 & $\begin{array}{l}00000 \\
11246\end{array}$ & 0.5 & $\begin{array}{l}00122 \\
22223\end{array}$ & 2 & $\begin{array}{l}00001 \\
11222\end{array}$ & 1 & $\begin{array}{l}00011 \\
22245\end{array}$ & 1.5 \\
\hline 15 & $\begin{array}{l}00012 \\
24457\end{array}$ & 2 & $\begin{array}{l}02222 \\
33357\end{array}$ & 2.5 & $\begin{array}{l}12223 \\
33344\end{array}$ & 3 & $\begin{array}{l}02333 \\
33444\end{array}$ & 3 & $\begin{array}{l}24445 \\
55666\end{array}$ & 3 \\
\hline 19 & $\begin{array}{l}11244 \\
45588\end{array}$ & 4 & $\begin{array}{l}02344 \\
45557\end{array}$ & 4 & $\begin{array}{l}24555 \\
66788\end{array}$ & 5.5 & $\begin{array}{l}25555 \\
66788\end{array}$ & 5.5 & $\begin{array}{l}46666 \\
67777\end{array}$ & 5.5 \\
\hline 23 & $\begin{array}{l}13333 \\
55788\end{array}$ & 4 & $\begin{array}{l}03344 \\
55567\end{array}$ & 4.5 & $\begin{array}{l}24555 \\
66888\end{array}$ & 5.5 & $\begin{array}{l}33344 \\
56668\end{array}$ & 4.5 & $\begin{array}{l}45555 \\
66678\end{array}$ & 4.5 \\
\hline 27 & $\begin{array}{l}12223 \\
36778\end{array}$ & 3 & $\begin{array}{l}02344 \\
44457\end{array}$ & 4 & $\begin{array}{l}23455 \\
55888\end{array}$ & 5 & $\begin{array}{l}33334 \\
55668\end{array}$ & 4.5 & $\begin{array}{l}44445 \\
55668\end{array}$ & 4.5 \\
\hline
\end{tabular}

Ten rats per group were injected with $0.5 \mu \mathrm{g} / \mathrm{mL}$ of each ultrafiltrate or $0.5 \mathrm{~mL}$ of Rifamycin SV ( $250 \mu \mathrm{mL})$ or saline solution at 7 and 8 days after arthritis induction $A A$ adjuvant arthritis, $A /$ arthritis induction, $R A$ rheumatoid arthritis

** $\mathrm{p}<0.05$ vs saline solution

Table 4 Protection of rats from adjuvant arthritis by administration of synovial fluid (UF-sf)

\begin{tabular}{llll}
\hline $\begin{array}{l}\text { Longlasting RA } \\
\text { synovial fluid } \\
(\mathbf{N}=\mathbf{8})\end{array}$ & $\begin{array}{l}\text { Recent RA } \\
\text { synovial fluid } \\
\mathbf{5 - 1 0} \text { days } \\
(\mathbf{N}=6)^{\mathbf{a}}\end{array}$ & $\begin{array}{l}\text { OA synovial } \\
\text { fluid }(\mathbf{N}=6)\end{array}$ & $\begin{array}{l}\text { Saline solution } \\
(\mathbf{N}=\mathbf{8})\end{array}$ \\
\hline $2.37 \pm 1.60^{\circ}$ & $5.9 \pm 1.01 \wedge$ & $6.7 \pm 095 \wedge$ & $7.5 \pm 0.92 \wedge$ \\
\hline
\end{tabular}

Clinical score at 21 days after arthritis induction

$R A$ rheumatoid arthritis, $O A$ arthrosis

${ }^{\circ} \mathrm{p}<0.01$ vs saline solution

${ }^{a}$ After a previous complete extraction

$\wedge$ Not significant. Each rat was treated with synovial fluid of an individual patient

APCs of PBMCs as well as in synovial cells. Recently, the presence of pathogenic-like $\mathrm{T}$ cells has been demonstrated in the blood stream of adult forms of autoimmune arthritis and juvenile chronic arthritis; these T cells share genotypic and phenotypic characteristics with synovial $\mathrm{T}$ cells [28]. Thus, the presence of pathogenic lymphocytes implies the presence of APCs as well.

The ultrafiltration of lysate and/or the suspension of cellular fragments immediately after centrifugation were intended to retain the proteolytic enzymes and allow numerous unknown small molecules, including the possible autoantigen, to pass into the ultrafiltrate aqueous solution that was used for subcutaneous injection. This extremely simplistic theory is supported by the development of an indisputable efficacy of the ultrafiltrates in patients with arthritis. Most notably, the therapeutic effect lasting 30 days from a subcutaneous dose is a type of response that differs completely from all known antiarthritic therapies [29].

In comparison, the administration of a specific, known autoantigen in appropriate doses, such as glutamic acid decarboxylate (GAD 65) in the non-obese diabetic rat, a well-known animal model of spontaneous autoimmune disease, has been shown to cure the disease [14].

In the present study, the two immunotherapeutic vaccines prepared from the rheumatoid PBMCs, UF- $f / t$ and UF-R, reached different outcomes in the two diseases; the UF-R was unable to modulate the animal model of arthritis but was effective in patients with RA while the UF-f/t worked both in AA of rats, reducing the severity of oncoming arthritis, and in patients with RA leading to an improvement of arthritic symptoms (Tables 1,5 ).

The other two ultrafiltrates, UF-S and UF-sf, exhibited immunotherapeutic activities comparable to that of

Table 5 Autologous immunotherapy of RA with ultrafiltrates derived from PBMCs and synovial fluids. The protection profile in AA is included

\begin{tabular}{llll}
\hline Lysate ultrafiltrates & Abbreviation & RA patients autologously injected & Adjuvant arthritis (AA) in rats iv injected \\
\hline Prepared from acellular synovial fluid & UF-sf & Active & Active \\
Prepared by freeze/thawing of PBMCs & UF-f/t & Active & Active \\
Prepared from supernatant of PBMCs culture & UF-S & ND & Active \\
Prepared by lysing PBMCs with rifamycin SV & UF-R & Active & Ineffective \\
\hline
\end{tabular}


the ultrafiltrate prepared by freeze/thawing of PBMCs (Table 5).

It is reasonable to assume that there are at least two unknown immunoactive epitopes, in both mononuclear cells and synovial fluid ultrafiltrates.

These epitopes are thrown out of disrupted cells, specially from their endosomal compartment, during the preparation of ultrafiltrates. They are not joined to any HLA DR molecule, they pass through an ultrafilter with a cut off of $10 \mathrm{kDa}$ (see "Methods" section) and are free of molecules involved in the endocytic processing of antigens (e.g., class II-associated invariant peptide).

The Rifamycin SV might have tied the epitope effective in AA, while preparing ultrafiltrate UF-R, but leaving intact the epitope effective in human arthritis. This results in the disabling of its property to protect rats from experimental arthritis.

The epitopes (two or more) of synovial fluid, spontaneously released following the lysis of proliferating synoviocites and deeper mononuclear cell of inflamed synovium, accumulate over time. If drawn out again, just a few days after a complete extraction of the effusion, this rheumatoid synovial fluid was ineffective on prevention of AA, unlike longlasting synovial fluid (Tables 4, 5). This might explain the previous reports on depressed proliferative response by PBMCs to mHsp60 in early RA [30]. The synovial fluids from arthrosis patients with arthrosis were always ineffective in modulating the AA of rats because they do not contain arthritogenic epitopes.

We know very little about the epitope effective in RA patients. It is present in both ultra filtrates of PBMCs and synovial fluid; it is effective as immunotherapeutic approach and exhibits immunomodulatory activities when is added to rheumatoid cell cultures (see below). We do not know the amino acid structure and the precise molecular weight. But It is just as small, much smaller than those identified with the pan-DR-binding Hspderived epitopes [31], as candidate for antigen specific bystander immunotherapy [32-35].

Once subcutaneously injected in RA patients, the epitope is transported by dendritic cells in inflammatory sites.

The status of the auto antigen in the ultrafiltrate, i.e., a peptidic fragment, not associated with a class II MHC molecule, might assist in explaining the early appearance of signs and symptoms of clinical improvement: being taken up and transported by dendritic cells, the selfepitope would likely interact directly with the antigen binding site of anti-idiotypic T cells on the cellular membrane (paratope), thus activating an immunoregulatory response. The idiotype-anti-idiotype network on the cell membrane is considered central in immunoregulation involving auto antigens. Therefore, the peptide would not interact with autoreactive $\mathrm{T}$ lymphocytes and thus would bypass the most detrimental component of the immune response in patients with arthritis. In fact, pro-inflammatory responses have never been observed in vivo or in vitro.

We hypothesized that the intrasynovial Rifamycin SV $[1,2]$, through these autonomic immunological mechanisms established an endogenous immunotherapy.

The PBMCs from RA patients, in baseline cultures, were unresponsive to synthetic HSP60 bystander peptide P1 (Fig. 1) but exhibited an immune response to the addition of the ultra filtrate UF-sf which was characterized by significant increase in the expression of CTLA- 4 by CD $4+C D 25+(p=0.05)$ and an equally significant reduction of CD69 $(\mathrm{p}=0.04)$; modifications which jointly imply an immunoregulatory response that only a specific antigen is able to elicit in subjects that have, in all probability, a basic immunological trend potentially oriented toward immunoregulatory responses.

In this context, a certain role might be played by the innate immunity that is persistently activated in patients with RA [36, 37].

The addition of autologous UF- $f / t$ to PBMCs cultured from vaccinated patients elicited minimal change in cellular markers (Table 2). This might indicate that mononuclear cells are functionally impaired by previous in vivo stimulation. In contrast, in cultures from normal subjects there was a marked reduction in the expression of CTLA-4 (-33\%) after the addition of autologous ultrafiltrate (Table 2). This finding reinforces the speculation that subjects with RA have a particular arthritic immunological profile, particularly earliness of clinical improvement and the duration of a single subcutaneous injection.

As regards the immune response in rats with ongoing arthritis, the mechanisms of protection might be the same with the difference that, being induced, the disease is self-limiting.

However, the epitope injected with human ultra filtrate, very likely related to HSP60 molecule, remains unknown. As the HSP is an extraordinarily evolutionarily conserved molecule the presence of its epitopes in the endocytic compartment of human cells is almost obviously. It is well known that $\mathrm{T}$ cells specific for self-Hsp even exist in the normal $\mathrm{T}$ cell repertoire.

Immune response to hsp60 by rheumatoid synovial cells and the presence of these antigens in inflamed joints and subcutaneous nodules of RA patients were reported $[38,39]$. In JCA this responsiveness was directed to self hsp60 and the increased response of Hsp60-specific 
T-cells correlated with the spontaneous disease remission [40].

Notably, the epitopes contained in our ultrafiltrates and responsible for the prevention of AA, appear to be smaller than the aa sequence $180-188$ of the mycobacterial HSP65 which, while preventing the AA and despite having therapeutic effects on this experimental model, is not considered as the pathogenic autoantigen (it is not able to induce the disease) [41].

The auto antigens extracted here, can be enriched many times $(\mathrm{w} / \mathrm{v})$, along with all the other small molecules, by aggregating multiple ultrafiltrates and subjecting them to lyophilization. Thus prepared, the ultrafiltrate might also be autologously used in non-rheumatological autoimmune disease, particularly in cases where the number of APCs in the blood is low, even if the autoantigen is unknown (personal obs). An adverse reaction to these antigens is unlikely given that peripheral lymphocytes reactive to self peptides are eliminated in the ontogenic selection.

In summary, this study outlines the unusual properties of the unknown and very probably pathogenic peptide that is contained in the ultrafiltrates prepared from rheumatoid PBMCs and synovial fluid. However, at the same time, the positive responses following subcutaneous administration of ultrafiltrates highlights the immunological profile of patients with RA, characterized by the potential capacity to reactivate, as a result of a specific stimulus, a pre-existing protective mechanism that is, of itself, insufficient to produce clinically significant effects.

The same human ultra filtrates contain an epitope, related to the molecule HSP60, that is able to modulate the immune mechanism implicated in AA and to stimulate protective immunity.

These studies should be considered very preliminary, and the clinical data must be interpreted with caution in view of the small number of cases.

No side effects were observed in our study following patient treatments, including at the site of subcutaneous administration of UF-sf or UF-f/t. However, on occasion the beneficial response was preceded by slight exacerbation of symptoms. Two patients in group UF-R experienced a hypersensitivity reaction after the second injection; both were characterized by skin eruptions that regressed spontaneously in a few days. In 13 patients, the intradermal sensitivity test to Rifamycin SV prior to enrollment was considered positive (appearance of wheal $\geq 0.5 \mathrm{~cm}$ ). None of our patients experienced side effects common to immunotherapies in humans and in most studied experimental models of autoimmune diseases such as diabetes and multiple sclerosis (hypersensitivity reactions, immunization towards cryptic epitopes, or de novo induction of autoimmune diseases).

\section{Conclusions}

- The disruption of PBMCs by the lytic action of Rifamycin or by freeze/thawing in vitro, caused the release of endosomal content and pathogenic autoantigens from APCs present in the circulation, which were then be isolated from degrading enzymes and bound immunologic molecules by ultrafiltration.

- The method of recovery, which utilize an ultrafilter with a cut-off of $10 \mathrm{kDa}$, lead to the conclusion that the possible pathogenic peptide is not associated with HLA-DR molecules.

- We prepared four different ultrafiltrates, two of which were derived from the breakdown of PBMCs, one from the supernatant of PBMCs cultures and one from synovial fluid. Three of these were utilized in trials of autologous immunotherapy by injecting them subcutaneously in 115 patients with early RA (disease duration of less than 12 months).

- At clinical monitoring on the 30th day, 22/58 (38 \%) patients treated with the autologous ultrafiltrate prepared by the freeze/thawing of PBMCs reached an ACR20. But in some patients these effects occurred early, sometimes after a week.

- The status of the auto antigen in the ultrafiltrate, i.e., a peptidic fragment, not associated with a class II MHC molecule, likely taken up and transported by dendritic cells, allows the administered pathogenic peptide to by-pass an interaction with autoreactive $\mathrm{T}$ cells and favor interaction with the anti-idiotype (paratope) antigen binding sites, activating an immunoregulatory response. The early appearance of clinical improvement in the patients might be related to this mechanism of response.

- Examining the results obtained in rats with AA and in subjects with RA it is reasonable to assume there are at least two unknown immunoactive epitopes: one, derived from the molecule of HSP60 (HSP is highly evolutionarily conserved molecule), which causes immunosuppression in rats, the other develops its immunotherapeutic capacity in RA patients. They are both present in humans.

- In vitro studies defined certain notable immunomodulatory properties of the ultrafiltrates, and thus of the peptide, and outlined a particular immunological profile of the subjects with RA.

- During the course of the immunotherapy with freeze/ thaw and acellular synovial fluid ultra filtrates, no side effects of any type were observed, nor were hypersensitivity or anaphylactic reactions induced. Adjuvant of any sort was never used. However, two subjects treated with ultrafiltrate prepared with Rifamycin SV experienced a morbilliform rash. 


\section{Perspectives}

In agreement with the preliminary results and considering the huge amount of knowledge in the field of human immunotherapy and in the prevention of experimental autoimmune diseases, we suggest that the use of ultrafiltrates, with the appropriate adjustment of the scheme, be applied for a perspective clinical study aimed at preventing the appearance of RA in subjects with a genetic profile associated with elevated risk for RA and/or a high level of anti-citrullinated protein antibodies. Furthermore, as autoantigen(s) can be enriched many times (w/v) through aggregation of multiple ultrafiltrates followed by lyophilization (Caruso unpublished), this method might also be applied in non-rheumatological autoimmune disease, particularly where the number of APCs in the blood is low

\section{Additional file}

Additional file 1. RIASSUNTO.

\section{Abbreviations}

UF-R: ultrafiltrate obtained by lysis of PBMCs with Rifamycin SV; UF-sf: ultrafiltrate obtained from acellular synovial fluid; UF-f/t: ultrafiltrate obtained by freeze/thaw of PBMCs; UF-S: ultrafiltrate obtained from supernatant of PBMC cultures.

\section{Authors' contributions}

CI wrote the paper, SP and SS carried out the study protocol and data analysis. GM and LA carried out animal experiments. SS, MF, CEM performed the analysis and interpretation of data. All authors read and approved the final manuscript.

\section{Author details}

1 Past Chief Rheumatology Unit, L. Sacco Hospital Milano Italy, Corso Italia 11 20122 Milan, Italy. ${ }^{2}$ Rheumatology UOC, L. Sacco Hospital Milano Italy, Milan, Italy. ${ }^{3}$ Director of microbiology chair, University of Milan, L. Sacco Hospital Milano Italy, Milan, Italy. ${ }^{4}$ Microbiology and Virology UO, L. Sacco Hospital milano Italy, Milan, Italy. ${ }^{5}$ Associazione Italiana Contro I'Artrite (AICA), Milan, Italy. ${ }^{6}$ Orthopedic Unit, Istituto Clinico San Siro, Milan, Italy. ${ }^{7}$ Chief of rheumatology Unit. L. Sacco Hospital Milano Italy, Milan, Italy.

\section{Acknowledgements}

We would like to thank Marco Cazzola MD Saronno (VA) for helpful discussion and proofreading the manuscript, Elissa Keogh and Angela Min, MD (Clinical Investigation Institute. University of California, San Diego) for assistance in laboratory assays.

\section{Competing interests}

The authors declare that the research was conducted in the absence of any competing interests. None of those who produce or market the Rifamycin SV had a role in the study design, interpretation of the data or the writing of manuscript.

Received: 3 February 2016 Accepted: 16 May 2016 Published online: 07 June 2016

\section{References}

1. Caruso I, Montrone F, Fumagalli M, Patrono C, Santandrea S, Gandini M. Rheumatoid knee synovitis successfully treated with intra-articular Rifamycin SV. Ann Rheum Dis. 1982;41:232-6.
2. Caruso I. Twenty years of experience with intra-articular Rifamycin for chronic arthritides. J Int Med Res. 1997;25:307-17.

3. Cottafava F, Pinelli G, Freschi PA, Nardelli E, Ginocchio L, Bertolotto M, Brida di Prio S. Sinovite reumatoide al ginocchio trattata con buon risultato con Rifamicina SV intrarticolare. Minerva Pediatr. 1982;34:33-7.

4. Lindblad S, Hedfors E, Malmsorg A. Rifamycin SV in local treatment of synovitis. A clinical, arthroscopic and pharmacologic evaluation. J Rheumatol. 1985;12:900-3.

5. Caruso I, Montrone F, Fumagalli M, Patrono C, Boselli P, Scrollini F. Rifamycin SV in the treatment of knee synovitis. Orthopedics. 1982;5:1336-43.

6. Marchesoni A, Sinigaglia L, Ranza R, Tosi S, Varenna M, Colombo B. Rifamycin SV versus triamcinolone in local treatment of rheumatoid synovitis. Scand J Rheumatol. 1993;22:194-8.

7. Blyth T, Stirling A, Coote J, Land D, Hunter A. Injection of the rheumatoid knee: Does intra-articular methotrexate or rifampicin add to the benefit of triamcinolone hexacetonide? Br J Rheumatol. 1998;37:770-2.

8. Caruso I, Cazzola M, Santandrea S. Clinical improvement in ankylosing spondylitis with rifamycin SV infiltrations of peripheral joints. J Int Med Res. 1992;20:171-81.

9. Caruso I, Santandrea S, Sarzi Puttini PC, Montrone F, Boccassini L, Bombaci A, Azzolini V, Cazzola M, Dell'Acqua D. Prevention of appearance of radiological lesions in early rheumatoid arthritis: a randomized, single-blind study comparing intra-articular rifamycin SV with auronofin. J Int Med Res. 1992;20:61-77.

10. Caruso I, Principi N, D'Urbino G, Santandrea S, Boccassini L, Montrone F, Sarzi Puttini PC, Bombaci A, Bozzato A, Azzolini V, Dell'Acqua D. Rifamycin SV administered by intra-articular infiltrations shows disease modifying activity in patients with pauici and polyarticular juvenile rheumatoid arthritis. J Int Med Res. 1993;21:243-56.

11. Jerne NK. Towards a network theory of the immune system. Ann Immunol. 1974;125C:373-89.

12. Hoffmann G. W: a network theory of the immune system. Eur J Immunol. 1975:5:638-47. doi:10.1002/eji.1830050912.

13. JC Cruse and RE Lewis. Atlas of immunology II Ed: Immunoglobulin synthesis CRC Press, 2004; (7): 173-225.

14. Tisch R, Wanb B, Serreze DV. Induction of glutamic acid decarboxylase 65 -specific Th2 cells and suppression of autoimmune diabetes at late stage of disease is epitope dependent. J Immunol. 1999;63:1178-87.

15. O'Neill SK, Shlomchik MJ, Glant TT, Cao Y, Doodes PD, Finnegan A. Antigen-specific B cells are required as APCs and autoantibody-producing cells for induction of severe autoimmune arthritis. J Immunol. 2005;174:3.

16. Roche PA, Furuta K. The Ins and outs ofd MHC class II-mediated antigen processing and presentation. Nat Rev Immunol. 2015;15:203-16. doi:10.1038/nri3818.

17. Sandberg JK, Glas R. Antigen processing USA Encyclopedia of Life Science 2001. Nature Public group.

18. Panay GS. B cells: a fundamental role in the pathogenesis of rheumatoid arthritis? Rheumatology (Oxford). 2005;44(Suppl 2):ii3-7.

19. O'Neill SK, Glant TT, Finnegan A. The Biscay role of B cells in animal models of rheumatoid arthritis. Front BioSci. 2007;12:1722-36.

20. Nakken B, Munthe LA, Konttinen YT, Sandberg AK, Szekanecz Z, Alex P, Szodoray P. B-cells and their targeting in rheumatoid arthritis-current concepts and future perspectives. Autoimmun Rev. 2011:11(1):28-34.

21. Van Eden W, Holoshitz J, Nevo Z, Frenkel A, Klajman A, Cohen IR. Arthritis induced by a T-lymphocyte clone that responds to Mycobacterium tuberculosis and to cartilage proteoglycan. Proc Natl Acad Sci USA. 1985;82:5117-20.

22. Gaston JSH, Life PF, Bailey LC, Bacon PA. In vitro responses to a 65-kilodalton mycobacterial protein by synovial T cells from inflammatory arthritis patients. J Immunol. 1989;143:2494-500.

23. Huang MN, Yu H, Moudgil KD. The involvement of heat-shock proteins in the pathogenesis of autoimmune arthritis: a critical appraisal. Semin Arthritis Rheum. 2010;40(2):164-75. doi:10.1016/j.semarthrit.2009.10.002.

24. Arnett FC, Edworthy SM, Bloch DA, McShane DJ, Fries JF, Cooper NS, et al. The American Rheumatism Association 1987 revised criteria for the classification of rheumatoid arthritis. Arthritis Rheum. 1988:31:315-24.

25. Hochberg MC, Chang RW, Dwosh I, Lindsey S, Pincus T, Wolfe F. The American College of Rheumatology 1991 revised criteria for the classification of global functional status in rheumatoid. Arthritis Rheum. 1992;35:498-502 
26. Felson DT, Anderson JJ, Boers M. American College of Rheumatology preliminary definition of improvement in rheumatoid arthritis. Arthritis Rheum. 1995:38:727-35.

27. Aquinas M, Allan WG, Horsfall PA, Jenkins PK, Hung-Yan W, Girling D, Tall R, Fox $\mathrm{W}$. Adverse reactions to daily and intermittent rifampicin regimens for pulmonary tuberculosis in Hong Kong. Br Med J. 1972;1 (5803):765-71.

28. Spreafico R, Rossetti M, van Loosdregt J, Wallace CA, Massa M, MagniManzoni S, Gattorno M, Martini A, Lovel DJ, Albani S: A circulating reservoir of pathogenic-like CD4+T cells shares a genetic and phenotypic signature with the inflamed synovial micro- environment. Ann Rheum Dis 2014;12. doi:10.1136/206226.

29. Weisman MH. Do all drugs and treatment for rheumatoid arthritis have the same efficacy? Nat Clin Pract Rheumatol. 2008;4(6):298-9. doi:10.1038/ncprheum0806.

30. Ramage JM, Gaston JS. Depressed proliferative response by peripheral blood mononuclear cells from early arthritis patients to mycobacterial heat shock protein 60. Rheumatology (Oxford). 1999;38:631-5.

31. de Yong H, Lafeber Floris FP, de Yager W, Haverkamp MH, Kuis W, Bijlsma Joanes WJ, Prakken BJ, Albani S. PAN-DR-binding hsp60 self epitopes induce an interleukin-10-mediated immune response in rheumatoid arthritis. Arthritis Rheum. 2009;60:1966-76.

32. Albani S, Prakken B. T cell epitope-specific immune therapy for rheumatic diseases. Arhtritis Rheum. 2006:54:19-25.

33. Prakken BJ, Samodal R, Le TD, Giannoni F, Yung GP, Scavulli J, Amox D, Roord S, de Kleer I, Bonnin D, Lanza P, Berry C, Massa M, Billetta R, Albani S. Epitope-specific immunotherapy induces immune deviation of proinflammatory $T$ cells in rheumatoid arthritis. Proc Natl Acad Sci USA. 2004;101(12):4228-33
34. Koffeman EC, Genovese M, Amox D, Keogh E, Santana E, Matteson EL, et al. Epitope-specific immunotherapy of rheumatoid arthritis. Arthritis Rheum. 2009:60:3207-16.

35. Zonneveld-Huijssoon E, van Wijk F, Roord S, Delemarre D, Meerding J, de Jager W, Klein M, Raz E, Albani S, Kuis W, Boes M, Prakken BJ. TLR9 agonist CpG enhances protective nasal HSP60 peptide vaccine efficacy in experimental autoimmune arthritis. Ann Rheum Dis. 2012;71:1706-15. doi:10.1136/annrheumdis-2011-201131.

36. Falgarone $\mathrm{G}$, Jaen $\mathrm{O}$. Boissier $\mathrm{MC}$ Role for innate immunity in rheumatoid arthritis. Joint Bone Spine. 2005;72(1):17-25.

37. Gierut A, Perlman H, Pope RM. Innate immunity and rheumatoid arthritis. Rheum Dis Clin North Am. 2010;36(2):271-96. doi:10.1016/j. rdc.2010.03.004.

38. Gaston JSH, Life PF, Bailey LC, Bacon PA. In vitro responses to a 65-kilodalton mycobacterial protein by synovial T cells from inflammatory arthritis patients. J Immunol. 1989;143:2494-500.

39. Klareskog L. Presence of human $65 \mathrm{kD}$ heat shock protein in inflamed joints and subcutaneous nodules of RA patients. Scand J Immunol. 1990;31:283-8

40. de Kleer IM, Kamphuis SM, Rijkers GT, Scholtens L, Gordon G, de Jager W, Hafner R, Zee R, van Eden W, Kuis W, Prakken BJ. The spontaneous remission of juvenile idiopathic arthritis is characterized by $C D 30+T$ cells directed to human heat-shock protein 60 capable of producing the regulatory cytokine interleukin-10. Arthritis Rheum. 2003;48:2001-10.

41. Yang X-D, Gasser J, Riniker B, Feige U. Treatment of adjuvant arthritis in rats: vaccination potential of a synthetic nonapeptide from the $65 \mathrm{kDa}$ heat shock protein of mycobacteria. J Autoimmun. 1990;3:11-23.

\section{Submit your next manuscript to BioMed Central and we will help you at every step:}

- We accept pre-submission inquiries

- Our selector tool helps you to find the most relevant journal

- We provide round the clock customer support

- Convenient online submission

- Thorough peer review

- Inclusion in PubMed and all major indexing services

- Maximum visibility for your research

Submit your manuscript at www.biomedcentral.com/submit
O Biomed Central 